\title{
INSERÇÃO DE GRADUANDOS DE ENFERMAGEM EM SERVIÇOS DE SAÚDE DO TRABALHADOR: RELATO DE EXPERIÊNCIA
}

\author{
Magda Guimarães de Araujo Fariaํㅜ, Cristiane Helena Gallasch², Amanda de Lucas Xavier Martins ${ }^{3}$
}

RESUMO: O objetivo desta pesquisa é relatar as ações realizadas no âmbito do campo prático da Saúde do Trabalhador no internato da Faculdade de Enfermagem da Universidade do Estado do Rio de Janeiro. A experiência foi vivenciada entre os meses de maio e dezembro de 2015 e os alunos participantes são discentes de Enfermagem do último ano. Foram criados três grupos de práticas, a citar: Participação nos programas de saúde do trabalhador da unidade; Educação em saúde para os trabalhadores; Análise de riscos ambientais de enfermarias específicas. Conclui-se que as atividades contribuíram para o desenvolvimento acadêmico, configurando-se como essencial à apropriação das competências básicas da respectiva área.

DESCRITORES: Enfermagem; Saúde do trabalhador; Programas de graduação em enfermagem; Enfermagem do Trabalho.

\section{INCLUSION OF NURSING UNDERGRADUATE STUDENTS IN OCCUPATIONAL HEALTH SERVICES: AN EXPERIENCE REPORT}

\begin{abstract}
The aim of this study was to report actions carried out in the practice field of occupational health in the boarding school of the School of Nursing of the Rio de Janeiro State University. The experience was carried out from May to December 2015 and the participants were senior nursing students. Three practice groups were formed as follows: Participation in the unit's occupational health programs; Education in occupational health; Analysis of environmental risks at specific wards. In conclusion, the activities contributed to academic development, and were considered essential for the learning of basic skills in the respective field. DESCRIPTORS: Nursing; Occupational Health; Education, Nursing, Diploma Programs; Occupational Health Nursing.
\end{abstract}

\section{INSERCIÓN DE ESTUDIANTES AVANZADOS DE ENFERMERÍA EN SERVICIOS DE SALUD LABORAL: RELATO DE EXPERIENCIA}

RESUMEN: El objetivo de esta investigación es informar las acciones realizadas en el ámbito del campo práctico de la Salud Laboral en la pasantía de la Facultad de Enfermería de la Universidad del Estado de Rio de Janeiro. La experiencia fue realizada entre los meses de mayo y diciembre de 2015, los alumnos participantes eran estudiantes del último año de Enfermería. Fueron creados tres grupos de prácticas, a saber: Participación en programas de salud laboral de la unidad; Educación en salud para los trabajadores; Análisis de riesgos ambientales de enfermerías específicas. Se concluye en que las actividades contribuyeron al desarrollo académico, configurándose como esenciales para la apropiación de las competencias básicas del área en cuestión.

DESCRIPTORES: Enfermería; Salud Laboral; Programas de Graduación en Enfermería; Enfermería del Trabajo.

${ }^{1}$ Enfermeira. Doutora em Enfermagem. Docente de Enfermagem da Faculdade de Enfermagem da Universidade do Estado do Rio de Janeiro. Rio de Janeiro, RJ, Brasil.

${ }^{2}$ Enfermeira. Doutora em Enfermagem. Docente de Enfermagem da Faculdade de Enfermagem da Universidade do Estado do Rio de Janeiro. Rio de Janeiro, RJ, Brasil.

${ }^{3}$ Enfermeira. Mestre em Educação Profissional em Saúde. Docente de Enfermagem da Faculdade de Enfermagem da Universidade do Estado do Rio de Janeiro. Rio de Janeiro, RJ, Brasil.

Autor Correspondente:

Magda Guimarães de Araujo Faria

Universidade do Estado do Rio de Janeiro

Av. Boulevard 28 de setembro 157 - 20551-030 - Vila Isabel, RJ, Brasil

E-mail: mag_araujo@yahoo.com.br
Recebido: $16 / 02 / 2016$ Finalizado: 07/11/2016 


\section{- INTRODUÇÃO}

A Saúde do Trabalhador compreende um campo teórico-prático da área da Saúde Coletiva, obtendo como eixo fundamental a relação do processo saúde-doença com o trabalho. No âmbito do Sistema Único de Saúde (SUS), a Política Nacional de Saúde do Trabalhador e Trabalhadora ${ }^{(1)}$ define diretrizes e estratégias para atenção integral em todos os pontos e instâncias da Rede de Atenção à Saúde (RAS), enfatizando a vigilância, a partir da promoção e proteção e redução da morbimortalidade. Considera a transversalidade das ações de saúde do trabalhador e o trabalho um dos determinantes do processo saúde e doença, assim como a proteção e prevenção de acidentes e agravos no ambiente de trabalho.

A enfermagem do trabalho emergiu no final do século XIX na Europa, com a revolução industrial, e teve sua consolidação no Brasil no final da década de 1970. A presença do enfermeiro na equipe de saúde do trabalhador estabeleceu suas práticas no ambiente de trabalho, de forma a prevenir o adoecimento, manter a produtividade e contribuir para a redução de custos com trabalhadores para as empresas ${ }^{(2)}$. Atualmente, a enfermagem demarca sua importância na área, atuando, por meio de equipes multiprofissionais, em cenários ainda pouco convencionais nos quais, por intermédio dos serviços de higiene, medicina e segurança do trabalho, influenciao perfil de saúde dos trabalhadores assistidos $^{(3-4)}$.

De acordo com uma proposta de currículo integrado, que apresenta aos discentes a vivência do enfermeiro em diversos campos, incluindo o da promoção à saúde do trabalhador - e consequente prevenção de agravos - no ambiente hospitalar, a Faculdade de Enfermagem da Universidade do Estado do Rio de Janeiro (FACENF-UERJ) insere seus alunos do último ano da graduaçãoneste contexto. $\mathrm{O}$ internato de enfermagemé composto, integralmente,durante dois semestres, de vivências práticas, nas quais os alunos podem aprimorar continuamente suas práticas assistenciais, gerenciais, educativas e de reflexão crítica, possibilitando o conhecimento e a atuação em diversos cenários da enfermagem ${ }^{(5)}$.

Especificamente na saúde do trabalhador, os alunos são inseridos no serviço de saúde do trabalhadorde um Hospital Universitário, o que contribui para que participem da rotina de trabalho da enfermeira do trabalho responsável, atuemnos programas de saúde já desenvolvidos na instituição e detectem novas demandas para futuras intervenções.

Neste contexto, o presente artigo foi construído com o objetivo de relatar as ações realizadas pelos internos de enfermagem da Faculdade de Enfermagem da Universidade do Estado do Rio de Janeiro, sob supervisão docente, no âmbito do campo prático da Saúde do Trabalhador.

\section{METODOLOGIA}

A experiência relatada ocorreu no internato de enfermagem, durante as atividades de Saúde do Trabalhador. Ressalta-se que, atualmente, este cenário de prática é contemplado no $8^{\circ}$ período da graduação, em um hospital estadual universitário do município do Rio de Janeiro.

A média de permanência é de 24 horas de atividades por grupo de cinco alunos. As ações são planejadas previamente, junto à enfermeira do trabalho do referido hospital. A realização das atividades ocorre sob supervisão docente.

Ao final do período de prática de cada grupo de alunos, foram realizadas oficinas avaliativas do campo. Essas oficinas consistiam em um momento de discussão entre alunos e a professora para a análise criteriosa das ações realizadas. As oficinas também eram a base para o fechamento de notas. Nesses espaços de discussão, era possível perceber a capacidade dos grupos em realizar a articulação entre os conteúdos teóricos da Saúde do Trabalhador e a prática, ou seja, programas institucionais e normas regulamentadas em caráter nacional.

O planejamento das atividades prevê a existência de uma homogeneidade de ações entre os diversos grupos de alunos; entretanto, demandas pontuais podem apontar a necessidade de intervenções específicas que fogem ao planejamento inicial. Durante a sistematização desta experiência, não houve nenhuma situação que causasse discrepância entre as experiências dos diversos grupos. 
Além disso, enfatiza-se que as discussões apresentadas a seguir resultam do processo avaliativo de ações sistematizadas, a partir da prática docente realizada entre os meses de maio e dezembro de 2015. Não são relatados casos individuais ou perfis de alunos, não sendo necessário, portanto, a assinatura do termo de consentimento livre e esclarecido. Os procedimentos respeitam a Resolução do Conselho Nacional de Saúde n466, de 12 de dezembro de $2012^{(6)}$.

\section{- RESULTADOS}

A Saúde do Trabalhador é um campo de especialidadedealguns cursos de ensino superior da grande área da saúde - dentre eles, aenfermagem.Dessa forma, a realização de práticas neste campo requer conhecimentos que são apresentados, gradativamente, aos alunos de graduação da Faculdade de Enfermagem da Universidade do Estado do Rio de Janeiro, desde o início do curso. Tais conhecimentos são ministrados em aulas teóricas e ações de biossegurança e ergonomia, inseridas em atividades assistenciais, que devem, entretanto progredir para uma visão crítica ampliada, ainda não dominada na chegada aos dois últimos semestres de graduação. Sendo assim, o primeiro grande desafio do internato em saúde do trabalhador foi proporcionar o embasamento teórico para a realização supervisionada das práticas específicas.

As atividades teóricas foram realizadas no próprio cenário de prática, com a apresentação de normas regulamentadoras específicas e da Política Nacional de Saúde do Trabalhador e da Trabalhadora. A metodologia utilizada foi a problematização, na qual os graduandos puderam associar o conteúdo ministrado com suas experiências anteriores, a partir de um diálogo contínuo e não hierarquizado.

Após a apresentação do aporte teórico, as vivências dos discentes de enfermagem no internato da saúde do trabalhador foram divididas em três momentos: 1) Participação nos programas de saúde do trabalhador da unidade; 2) Educação em saúde com os trabalhadores; 3) Análise de riscos ambientais de enfermarias específicas.

Em relação a participação do discentes em programas de saúde do trabalhador da unidade, o Programa de Tuberculose Ocupacional (PTO) foi contemplado com ações discentes durante todo o período, a fim de detectar precocemente portadores de sintomas respiratórios relacionados ao acometimento pela tuberculose.

O foco do PTO é evitar o adoecimento dos trabalhadores, uma vez que um único caso pode se desdobrar em inúmeras contaminações. Ressalta-se que o programa também cumpre um importante papel educativo na prática profissional dos trabalhadores envolvidos, visto que, a cada busca, são esclarecidas dúvidas que auxiliam no entendimento acerca da tuberculose, podendo ser considerado um importante dispositivo de controle epidemiológico.

A inserção dos discentes no PTO ocorreu através da busca ativa semanal de pacientes, familiares ou trabalhadores com sintomas sugestivos à infecção pela tuberculose respiratória, em enfermarias selecionadas do hospital. Ressalta-se que todos os setores do Hospital Universitário são avaliados semanalmente; entretanto, devido a restrições temporais, os estudantes do internato de enfermagem responsabilizaram-se por algumas enfermarias selecionadas pelo serviço de saúde do trabalhador do local.

No início, a busca realizada no âmbito do PTO gerou dúvidas das equipes de enfermagem quanto à relevância da atividade. Entretanto, com o passar do tempo - e com as orientações semanais dos discentes sobre a necessidade do controle da tuberculose -, observou-se não só uma modificação na receptividade desta atividade, como também um aumento no relato de casos suspeitos.

Em alguns setores analisados, o aumento no número de casos suspeitos foi superior a 50\%; entretanto, não houve modificações nos números absolutos de sujeitos com tuberculose. A atuação discente nas situações de possibilidade de acometimento da tuberculose variava de acordo com o tipo de suspeita. Em casos cuja presunção de adoecimento é voltada para um paciente, o procedimento dos alunos restringia-se à coleta de dados do prontuário e acompanhamento do resultado dos exames diagnósticos. Quando se tratava de familiares ou visitantes, a atuação discente compreendia, basicamente, a educação em saúde. Já em situações que envolviam profissionais do hospital, a 
atividade discente abrangia uma criteriosa avaliação sintomática, transcrição de informações para o serviço especializado, encaminhamento imediato do trabalhador e o acompanhamento do caso.

O constante contato com os profissionais das enfermarias, investigadas pelo grupo dentro do PTO, acarretou o levantamento de outras questões que geravam dúvidas às equipes, o que suscitou na elaboração do segundo grupo de atividades discentes: A educação em saúde com os trabalhadores.

A partir dos questionamentos dos profissionais de saúde e considerando a dificuldade para a realização de atividades de educação permanente com os profissionais das enfermarias, devido à rotina de trabalho, o grupo construiu cartazes sobre acondicionamento do lixo e utilização de equipamentos de proteção individual. O objetivo traçado pelos alunos foi de que esse material fosse um estímulo para reflexão dos profissionais da enfermagem em relação aos problemas na rotina, e que fosse a consolidação do elo educativo com as unidades de internação, de forma a abrir espaço de diálogo e estabelecer uma parceria sólida neste processo de qualificação.

A aproximação da equipe do internato com as enfermarias atendidas pelo PTO, aliada à entrega do material educativo, culminou na parceria entre a equipe e um setor especializado do hospital para a realização de atividades de educação permanente. Inicialmente, a proposta era de realização de um momento educativo com a equipe técnica de enfermagem, cuja abordagem seria voltada para o "Uso de equipamentos de proteção individual durante os procedimentos na enfermaria". Contudo, a ação foi estendida por mais algumas semanas, para que todas as equipes atuantes no setor pudessem ser contempladas com a atividade. Além disso, as residentes de enfermagem também foram incluídas no público-alvo. Os grupos de alunos envolvidos planejaram, programaram e executaram a atividade sob supervisão docente direta.

A dinâmica realizada nesta atividade foi a roda de conversa, associada a um quiz de perguntas e respostas sobre a temática principal. A proposta era que a atividade não fosse apenas um momento formal de educação, mas também uma possibilidade de criação de vínculo, já que toda discussão gerada foi embasada na própria experiência do público-alvo. Além disso, foi produzido material impresso para futuras consultas dos profissionais daquele setor.

Finalmente, como terceiro grupo de atividades desenvolvidas pelos discentes foi realizada a análise de riscos ambientais de enfermarias específicas. A atividade de análise de riscos ambientais realizada com os discentes do curso de graduação em enfermagem pode ser considerada um ensaio na construção de um PPRA. Isto porque, tendo em vista a rotatividade dos alunos, não há tempo suficiente para a realização de todas as etapas envolvidas neste processo, incluindo: planejamento anual com estabelecimento de metas traçadas junto à equipe multiprofissional, prioridades e cronograma; estratégia e metodologia de ação; forma do registro, manutenção e divulgação dos dados; periodicidade e forma de avaliação do desenvolvimento do PPRA.

O objetivo desta atividade foi demonstrar aos discentes os riscos a que os trabalhadores estão expostos dentro de enfermarias específicas da instituição, como por exemplo, a nefrologia, a pediatria, a clínica médica e a Unidade de Terapia Intensiva (UTI), tornando-os capazes de analisar outros ambientes de atuação sob uma ótica reflexiva e resolutiva, intervindo assim, em situações de risco já naturalizadas nos espaços laborais.

Neste sentido, a primeira etapa para a realização da atividade foi a escolha de espaços disponíveis para análise. Foram eleitas três unidades, cuja inserção da equipe de internato foi feita a partir da sensibilização dos enfermeiros responsáveis por tais setores. Ressalta-se que não houve nenhum tipo de resistência ou tensão das equipes de trabalho.

A segunda etapa consistiu na identificação dos grupos de risco a serem avaliados. A referida avaliação verifica o potencial de danos à saúde causados pela exposição de indivíduos a agentes ambientais.A terceira etapa foi a de tabulação dos riscos encontrados. A quantificação destes riscos resultou em cinco riscos físicos; sete, químicos; 14, biológicos; três, ergonômicos e quatro riscos acidentais. Ressalta-se que estes riscos foram observados em diversos ambientes do cenário analisado, porém quantificados uma única vez.

A quarta etapa deste processo foi voltada para as intervenções discentes. Tendo em vista a restrição temporal, os alunos do internato de enfermagem realizaram apenas intervenções educativas, ou seja, 
agiram baseados em riscos causados, sobretudo, pelo comportamento humano, como, por exemplo, uso de adornos em ambientes de trabalho.

Ao final desta atividade, foi elaborado um relatório com todos os riscos encontrados e sugestões para sua possível minimização. Este material foi entregue para o núcleo de saúde do trabalhador da unidade em formato impresso e digital. A divulgação aos setores analisados foi realizada em formato digital pelo próprio núcleo de saúde do trabalhador.

\section{DISCUSSÃO}

Já é reconhecido que o envolvimento precoce do estudante com as questões do ambiente de trabalho nas aulas práticas e nos estágios propicia o desenvolvimento de ações voltadas à própria saúde e à possibilidade de reproduzir essas ações quando trabalhador e coordenador de ações de saúde $^{(7)}$.

Nesse sentido, a formação do aluno necessita ser pautada em propostas metodológicas que permitam o crescimento crítico e intelectual, como a problematização. As ações baseadas nesta perspectivapermite os estudantes desenvolverem reflexões críticas do conhecimento científico a partir de situações-problema das práticas cotidianas, que envolvem os sujeitos, modo de vida, relações sociais e o mundo do trabalho, articulando soluções estratégicas coletivas ${ }^{(8-9)}$.

A participação do discentes em programas de saúde do trabalhador da unidade articula os objetivos do PTO com as medidas propostas pelo Ministério da Saúde para o controle da tuberculose no Brasil, a citar: 1. Otimizar a busca por sintomáticos respiratórios; 2. Estabelecer um fluxo eficaz de atendimento aos sintomáticos respiratórios, priorizando o diagnóstico e a inicialização do tratamento; 3. Efetuar práticas de vigilância em saúde ${ }^{(10)}$.

A realização das atividades educativas propostas pelos discentes obedeceu a estrutura teóricometodológica da Educação Permanente em Saúde. De acordo com a Política Nacional de Educação Permanente em Saúde ${ }^{(11)}$, a educação permanente consiste na aprendizagem durante o exercício do trabalho. Tal processo permite a transformação das práticas profissionais e organização do trabalho, as quais acontecem no cotidiano das pessoas e das organizações, baseadas nos problemas enfrentados e nos conhecimentos e experiências dos indivíduos envolvidos.

A proposta de educação permanente em saúde, na perspectiva de transformação,é uma alternativa para os métodos tradicionais de educação, com articulação entre a teoria e prática realizada pelos sujeitos-trabalhadores, contextualizadas histórica, social, econômica, política e eticamente ${ }^{(12)}$.

Sobre o levantamento de riscos à saúde, a legislação é constituída por uma série de instrumentos que auxiliam o enfermeiro do trabalho na manutenção do estado de saúde dos trabalhadores. Um dos principais dispositivos utilizados é a avaliação de riscos ambientais à saúde, prevista na Norma Regulamentadora de $n^{\circ}$ 9, de 29 de dezembro de 1994, e é formalmente identificada como Programa de Prevenção de Riscos Ambientais (PPRA). Seu principal objetivo é a manutenção da saúde dos trabalhadores, através do reconhecimento e intervenções associados a possíveis riscos ambientais em espaço laboral|(13).

Nesse sentido, optou-se por utilizar a classificação dos riscos em: químicos, físicos, biológicos, ergonômicos e acidentais ${ }^{(14)}$ nos quais evidenciou-se a associação com a Norma Regulamentadora de $n^{\circ}$ 32, de 30 de agosto 2011, cuja temática central é a "Segurança e saúde no trabalho em serviços de saúde"(15).

\section{CONCLUSÃO}

O conjunto de atividades realizadas no internato em saúde do trabalhador visa proporcionar ao aluno uma primeira experiência nesta área de especialidade, sensibilizando-os a refletir sobre os processos de adoecimento no trabalho e a naturalização dos riscos à saúde em espaço hospitalar.

A realização das ações descritas colaborou para estimular um olhar crítico e reflexivo dos internos 
frente às inadequações presentes no ambiente laboral, sob perspectiva ética e legal do exercício profissional e da adequação à legislação brasileira relacionada à saúde no trabalho, alémde proporcionar maior clareza sobre o processo de trabalho em saúde do trabalhador.

É possível afirmar que as experiências vivenciadas pelos graduandos de enfermagem serão valiosas para suas vidas profissionais, já que não visavam apenas à capacidade técnica, mas também à reflexão sobre o cuidar.

Estima-se que este relato de experiência possa sensibilizar docentes de instituições de ensino de graduação em enfermagem sobre a importância destas atividades para a formação técnica, científica e social do enfermeiro. Afinal, a oferta de ações em saúde do trabalhador contribui grandemente para a promoção da saúde e para o incremento na qualidade de vida no trabalho, diminuindo o número de adoecimentos e afastamentos.

\section{REFERÊNCIAS}

1. Ministério da Saúde (BR). Portaria n. 1823, de 23 de agosto de 2012. Instituí a Política Nacional da Saúde do Trabalhador e Trabalhadora. Diário Oficial da República Federativa do Brasil, Brasília, 24 ago. 2012.

2. de Mendonça HPF, da Silva SEV. Reflexão crítica sobre a gênese da enfermagem do trabalho. Revenferm UFPEonline. [Internet] 2015;9(Suppl.7) [acesso em 02 ago 2016]. Disponível: http://www.revista.ufpe.br/ revistaenfermagem/index.php/revista/article/view/7747.

3. Paz PO, Kaiser DE. A busca pela formação especializada em enfermagem do trabalho por enfermeiros.Rev. Gaúcha Enferm.[Internet] 2011;32(1) [acesso em 13 mar 2016]. Disponível: http://dx.doi.org/10.1590/S198314472011000100003.

4. Lino MM, Nora PT, Lino MM, Furtado M. Enfermagem do trabalho à luz da visão interdisciplinar. Saúde Transform. Soc. [Internet] 2012;3(1) [acesso em 5ago 2016]. Disponível:http://pepsic.bvsalud.org/scielo.php?script=sci_artte xt\&pid=S2178-70852012000100014.

5. Faculdade de Enfermagem da Universidade do Estado do Rio de Janeiro. Sobre o internato de enfermagem. [Internet] 1 tela [acesso em 13 mar 2016]. Disponível:http://www.facenf.uerj.br/index.php/graduacao/internatode-enfermagem.

6. Ministério da Saúde (BR). Conselho Nacional de Saúde. Diretrizes e normas regulamentadoras de pesquisa envolvendo seres humanos. Resolução n. 466, de 12 de dezembro de 2012. Brasília; 2012.

7.Azambuja EP, Kerber NPC, Kirchhof AL. A saúde do trabalhador na concepção de acadêmicos de enfermagem. Rev.esc. enferm. USP. [Internet] 2007;41(3) [acesso em 31 ago 2016]. Disponível: http://dx.doi.org/10.1590/S008062342007000300003.

8. da Silva WB, Delizocov D. Problemas e problematizações: implicações para o ensino dos profissionais da saúde. Ensino, Saúde e Ambiente. [Internet] 2008;1(2) [acesso em 31 ago 2016].Disponível http://ltc-ead.nutes. ufrj.br/constructore/objetos/abp1.pdf.

9. Strochein KA, Zocche DAA. Educação permanente nos serviços de saúde: um estudo sobre as experiências realizadas no Brasil. Trab.educ.saúde. [Internet] 2011;9(3) [acesso em 31 ago 2016]. Disponível:http://dx.doi. org/10.1590/S1981-77462011000300009.

10. Ministério da Saúde (BR). Secretaria de Vigilância em Saúde. Departamento de Vigilância Epidemiológica. Manual de recomendações para o controle da tuberculose no Brasil. Brasília: Ministério da Saúde; 2011.

11. Ministério da Saúde (BR).Portaria n. 278, de 27 de fevereiro de 2014. Institui diretrizes para implementação da Política de Educação Permanente em Saúde, no âmbito do Ministério da Saúde (MS). Diário Oficial da República Federativa do Brasil, Brasília, 28 fev. 2014.

12. Sade PMC, Peres AM, Pedroso JVM, Larocca LM. Núcleo de enfermeiros de educação permanente do Paraná: trajetória e contribuições. CogitareEnferm. [Internet] 2016;21(2) [acesso em 05ago 2016]. Disponível:http://dx.doi. org/10.5380/ce.v21i2.44335. 
13. Ministério do Trabalho e Emprego (BR). Portaria MTE n. 1.471, de 24 de setembro de 2014. Altera o texto da Portaria no 25, de 29 de dezembro de 1994 Norma Regulamentadora n 9 - Riscos Ambientais. Diário Oficial da República Federativa do Brasil, Brasília, 25 set 2014.

14. Souza e Souza L, Pinto IS, Diamantino NAM, Franco EFPM, Gonçalves EC, Moura e Silva W, Santana JMF. Mapeamento dos riscos ambientais do laboratório de análises clínicas de um hospital de ensino: relato de experiência. G\&S. [Internet] 2013;4(1) [acesso em 05ago 2016]. Disponível: http://gestaoesaude.unb.br/index. php/gestaoesaude/article/view/332.

15. Ministério do Trabalho e Emprego (BR). Portaria nº 1748, de 30 de agosto de 2011. Institui o Plano de Prevenção de Riscos de Acidentes com Materiais Pérfurocortantes e altera a Norma Regulamentadora n. 32, que trata da segurança e saúde no trabalho em estabelecimentos de saúde. Diário Oficial da República Federativa do Brasil, Brasília, 31 ago. 2011. 